\title{
Dialysis Disequilibrium Syndrome and Cerebellar Herniation with Successful Reversal Using Mannitol
}

\author{
Anna Curtis (D), ${ }^{1}$ Christian Lamb $\mathbb{D}^{1},{ }^{2}$ Hussain Rao, ${ }^{1}$ Andrew Williams, ${ }^{1}$ and Amit Patel ${ }^{3}$ \\ ${ }^{1}$ School of Medicine, University of Missouri-Kansas City, Kansas City, MO 64108, USA \\ ${ }^{2}$ Department of Medicine, Brooke Army Medical Center, San Antonio, TX 78234, USA \\ ${ }^{3}$ Department of Medicine-Division of Nephrology, Truman Medical Center, Kansas City, MO 64108, USA
}

Correspondence should be addressed to Anna Curtis; akc8bv@mail.umkc.edu and Christian Lamb; christian.lambmd@gmail.com

Received 23 April 2020; Revised 23 July 2020; Accepted 30 July 2020; Published 25 August 2020

Academic Editor: Manel Vera

Copyright (C) 2020 Anna Curtis et al. This is an open access article distributed under the Creative Commons Attribution License, which permits unrestricted use, distribution, and reproduction in any medium, provided the original work is properly cited.

Dialysis disequilibrium syndrome (DDS) is a morbid but rare complication of dialysis. Feared sequalae of this diagnosis are brain herniation and death. This report presents a patient who was diagnosed with DDS with subsequent tonsillar herniation shown on imaging with complete resolution of clinical signs and symptoms, as well as imaging findings of herniation after prompt initiation of intravenous mannitol. This is the first known case of reversal and survival of DDS-induced tonsillar herniation using mannitol.

\section{Introduction}

Dialysis disequilibrium syndrome (DDS) is a neurological complication of hemodialysis typically seen in individuals receiving therapy for the first time. First described in 1962, symptoms can include mild headache, vomiting, altered sensorium, seizures, arrhythmias, and even death [1]. Current research suggests that the reverse urea effect may be the primary mechanism due to which this syndrome occurs. Urea and other osmotically active solutes are critical in maintaining intravascular volume. During hemodialysis, these intravascular solutes are removed rapidly, creating an osmotic gradient that favors free water influx into the cerebral parenchyma [2]. This brisk expansion of tissue compresses pivotal brain structures and can result in cerebral herniation.

The diagnosis of DDS is one of the exclusions and may be difficult to identify by clinicians due to its vast array of presenting symptoms [3]. Luckily, most cases of DDS are mild and self-resolving, though a myriad of devastating consequences have been reported, including demyelination, posterior reversible encephalopathy syndrome, and most notably, cerebral herniation [4-6]. An extensive literature review yielded thirteen documented reports of patients suffering herniation secondary to DDS with poor outcomes.
We present what we believe to be the first reported case of cerebellar tonsillar herniation secondary to DDS which was resolved using intravenous (IV) mannitol with no residual neurologic deficits.

\section{Case Presentation}

A 47-year-old African-American male with hypertension presented to the emergency department (ED) with complaints of persistent abdominal pain. Upon evaluation, the patient described his pain as vague and periumbilical. $\mathrm{He}$ also reported episodes of nonbloody emesis, headache, and possible hematuria since awaking that morning. His vital signs were significant for a blood pressure of $240 / 161 \mathrm{mmHg}$, heart rate of 100 beats per minute, respiratory rate of 18 breaths per minute, and oxygen saturation of $100 \%$ while he was breathing ambient air. He was afebrile. Physical exam revealed a malnourished-appearing male with an otherwise normal physical exam. Initial laboratory studies were significant for a creatinine of $15.39 \mathrm{mg} / \mathrm{dL}$ (reference range $0.90-1.3 \mathrm{mg} / \mathrm{dL}$ ), blood-urea-nitrogen (BUN) of $155 \mathrm{mg} / \mathrm{dL}$ (reference range $8-20 \mathrm{mg} / \mathrm{dL}$ ), an anion gap metabolic acidosis of $29 \mathrm{mmol} / \mathrm{L}$ (reference range $10-20 \mathrm{mmol} / \mathrm{L}$ ), platelet count of $4510^{3} / \mathrm{cmm}$ (reference range $150-40010^{3} /$ $\mathrm{cmm}$ ), and hemoglobin of $10.1 \mathrm{~g} / \mathrm{dL}$ (reference range 
$14.0-18.0 \mathrm{~g} / \mathrm{dL})$. Abdominal and pelvic computed tomography (CT) without contrast was performed and showed enteritis involving the jejunum.

The patient denied any history of renal or hematological disease. His home medications included labetalol $30 \mathrm{mg}$ once per day and nifedipine $100 \mathrm{mg}$ twice per day, both of which he admitted using sparingly. The patient was given intravenous (IV) labetalol in the ED with minimal success in lowering the blood pressure. Due to the presence of endorgan damage, hematological and metabolic aberrancies, thrombotic consumptive coagulopathy secondary to malignant hypertension was suspected. The patient was urgently admitted to the Intensive Care Unit (ICU) for further investigation and intervention.

In the ICU, a continuous IV infusion of nicardipine was initiated. Clinicians recommended urgent placement of a central venous catheter in order to initiate hemodialysis given electrolyte abnormalities. Initially, the patient refused all interventions due to holistic reasons. Fifteen hours following admission, the patient continued to deteriorate with worsening thrombocytopenia and encephalopathy; he ultimately agreed to catheter placement. Two units of platelets were transfused prior to the procedure. A right internal jugular vein triple lumen catheter was placed without complication, and the patient was initiated on hemodialysis with the dialysis prescription shown in Table 1.

A low-flux urea membrane was initially ordered by the attending nephrologist, but this option was not available at the facility in which this patient received treatment. Due to the urgency of treatment, hemodialysis was performed without this membrane.

Approximately 20 minutes into the hemodialysis session, the patient became unresponsive. A right eye gaze deviation and right-sided fasciculations of the upper and lower extremities were observed. Hemodialysis was discontinued, and an urgent head CT without contrast was performed. Imaging revealed pontine edema with mass effect and cerebellar tonsillar herniation (Figure 1). The on-call nephrologist initiated intravenous mannitol, and neurosurgery was consulted urgently. The results of complete basic metabolic panels before and after the initiation of hemodialysis are shown in Table 2.

Within 30 minutes of initiation of the mannitol infusion, the patient regained consciousness and remained hemodynamically stable. Follow-up neurological exam showed no focal deficits and complete resolution of the right-sided fasciculations and gaze palsy. Mannitol was continued for 24 hours. The following morning, head magnetic resonate imaging (MRI) was obtained and showed resolution of the midline shift and tonsillar herniation shown on the head CT scan eight hours prior. A diagnosis of Dialysis Disequilibrium Syndrome was made.

Three days following this diagnosis, a low-flux urea filter was obtained from an outside facility and the patient began continuous renal replacement therapy (CRRT). Serial head CT occurring every 48 hours and scheduled hourly neurologic examinations confirmed the patient's tolerance of CRRT with no further progression or recurrence of symptoms related to DDS. The patient was eventually transferred
TABLE 1: Dialysis prescription.

\begin{tabular}{lc}
\hline $\begin{array}{l}\text { F180 membrane } \\
\text { Surface area }\end{array}$ & $1.7 \mathrm{~m}^{2}$ \\
KUf & $58 \mathrm{~mL} / \mathrm{hr} / \mathrm{mmHg}$ \\
\hline Dialysate solution & \\
$\quad$ Sodium & $140 \mathrm{mmol} / \mathrm{L}$ \\
Potassium & $4 \mathrm{mmol} / \mathrm{L}$ \\
Calcium & $2.5 \mathrm{mmol} / \mathrm{L}$ \\
$\quad$ Bicarbonate & $35 \mathrm{mmol} / \mathrm{L}$ \\
\hline $\mathrm{Q}_{\mathrm{D}}$ & $600 \mathrm{~mL} / \mathrm{min}$ \\
\hline $\mathrm{Q}_{\mathrm{B}}$ & $300 \mathrm{~mL} / \mathrm{min}$ \\
\hline
\end{tabular}

out of the ICU and underwent placement of a long-term hemodialysis fistula. He had complete resolution of symptoms at the 2-year follow-up, but remained on intermittent hemodialysis.

\section{Discussion}

While the mechanism of DDS remains unclear, several risk factors for the syndrome have been proposed, including first dialysis, extremes of age, electrolyte imbalances, metabolic acidosis, hypertensive emergency, pre-existing neurological conditions, cerebral edema, meningitis, and the presence of intracranial tumors [7]. Additionally, the hemodialysis prescription prescribed by the provider may influence a fluid shift into the cerebral parenchyma. Current guidelines recommend limiting the removal of BUN to $40 \%$ over the course of two hours. This can be achieved by utilizing lowflux dialysis membranes, setting low flow rates (Qd of 300 and $\mathrm{Qb} 250$, respectively), modulating the dialysate sodium and glucose concentrations, and shortening the duration of therapy. Additional methods, including prophylactic mannitol administration, have been suggested $[2,7,8]$.

As described in the case above, our patient had several predisposing risk factors for DDS upon presentation to the ED. He was in hypertensive emergency and had a metabolic acidosis and significantly elevated BUN. This was also his first-time dialyzing. When dialysis was initiated, the duration was shortened to 120 minutes, dialysate sodium was set at $140 \mathrm{mmol} / \mathrm{L}$, and there was an attempt to secure a low-flux dialysis membrane. Unfortunately, even with these adjustments to therapy, the patient cleared $31 \mathrm{mg} / \mathrm{dL}$ of BUN or $20 \%$ of his total serum BUN in 20 minutes, as seen by comparing the patient's initial metabolic panel to the one drawn thirty minutes after initiation of HD (Table 2). Other interventions, such as the addition of dialysate glucose, increasing dialysate sodium further, utilizing lower $\mathrm{Qb}$ and Qd flow rates, and administering prophylactic mannitol, may have been beneficial at reducing the likelihood of DDS occurrence in this patient.

Historically, even with swift intervention, cerebral herniation due to DDS carries a grim prognosis. A case series by Osgood et al. in 2015 highlighted that four patients who all received hemodialysis were diagnosed with DDS and had cerebral herniation confirmed on imaging. Despite intervention with mannitol and hypertonic saline, all patients succumbed to their neurologic injury and were pronounced brain dead [6]. Similarly, a case series published by Kumar 


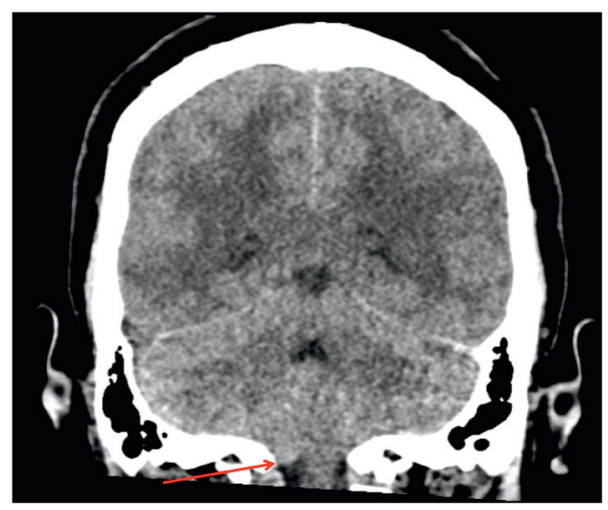

FIgure 1: Computed Tomography (CT) showing cerebellar tonsil herniation (red arrow).

TABLE 2: Results of basic metabolic panels.

\begin{tabular}{lcr}
\hline & Predialysis & Postdialysis \\
\hline Sodium & $135 \mathrm{mmol} / \mathrm{L}$ & $137 \mathrm{mmol} / \mathrm{L}$ \\
Potassium & $3.2 \mathrm{mmol} / \mathrm{L}$ & $3.2 \mathrm{mmol} / \mathrm{L}$ \\
Chloride & $89 \mathrm{mmol} / \mathrm{L}$ & $91 \mathrm{mmol} / \mathrm{L}$ \\
Bicarbonate & $22 \mathrm{mmol} / \mathrm{L}$ & $26 \mathrm{mmol} / \mathrm{L}$ \\
Blood-Urea-Nitrogen & $152 \mathrm{mg} / \mathrm{dL}$ & $121 \mathrm{mg} / \mathrm{dL}$ \\
Creatinine & $16.25 \mathrm{mg} / \mathrm{dL}$ & $11.75 \mathrm{mg} / \mathrm{dL}$ \\
Glucose & $175 \mathrm{mg} / \mathrm{dL}$ & $130 \mathrm{mg} / \mathrm{dL}$ \\
\hline
\end{tabular}

et al. in 2014 highlighted a patient suffering from DDS with resultant tonsillar herniation. Despite timely introduction of an infusion of hypertonic saline, the patient died [9]. With the exception of the case reported above, an extensive literature review failed to reveal any other documented cases of survival (with or without return to baseline) among patients with DDS and imaging-confirmed tonsillar herniation.

Guideline-based management of cerebral herniation secondary to DDS currently recommends the cessation of $\mathrm{HD}$, hyperventilation, and elevating the head of the bed. Although numerous studies have shown mannitol's efficacy at treating a variety of neurological conditions, the use of mannitol and other osmotically active infusions for the management of DDS remains controversial [10,11]. This report is the first known case in which a mannitol infusion successfully reversed DDS-induced tonsillar herniation with a complete return to neurologic baseline.

\section{Conclusions}

The case reported herein is the first documented account of reversal of the clinical and imaging findings of tonsillar herniation secondary to DDS using intravenous mannitol.

\section{Conflicts of Interest}

The authors declare no conflicts of interest. The views expressed herein do not reflect the official policy or position of the Brooke Army Medical Center, the U.S. Army Medical Department, the U.S. Army Office of the Surgeon General, the Department of Defense or the Departments of the Army, Navy, or Air Force, or the U.S. Government.

\section{References}

[1] A. C. Kennedy, A. L. Linton, and J. C. Eaton, "Urea levels in cerebrospinal fluid after hæmodialysis," The Lancet, vol. 279, no. 7226, pp. 410-411, 1962.

[2] N. Patel, P. Dalal, and M. Panesar, "Fellows' forum fellows' forum in dialysis edited by Mark A. Perazella: dialysis disequilibrium syndrome: a narrative review," Seminars in Dialysis, vol. 21, no. 5, pp. 493-498, 2008.

[3] A. Muzzammil and U. Bakhsh, "A vanishing complication of haemodialysis: dialysis disequilibrium syndrome," Journal of the Intensive Care Society, vol. 21, no. 1, pp. 92-95, 2018.

[4] A. Soomro, R. Al Bahri, N. Alhassan, F. Hejaili, and A. Al Sayyari, "Posterior reversible encephalopathy syndrome with tactile hallucinations secondary to dialysis disequilibrium syndrome," Saudi Journal of Kidney Diseases and Transplantation: An Official Publication of the Saudi Center for Organ Transplantation, Saudi Arabia, vol. 25, no. 3, pp. 625-629, 2014.

[5] S. C. Keswani, "Central pontine and extrapontine myelinolysis owing to disequilibrium syndrome," Journal of Child Neurology, vol. 19, no. 1, pp. 79-80, 2004.

[6] M. Osgood, R. Compton, R. Carandang, W. Hall, G. Kershaw, and S. Muehlschlegel, "Rapid unexpected brain herniation in association with renal replacement therapy in acute brain injury: caution in the neurocritical care unit," Neurocritical Care, vol. 22, no. 2, pp. 176-183, 2014.

[7] K. Mistry, "Dialysis disequilibrium syndrome prevention and management," International Journal of Nephrology and Renovascular Disease, vol. 12, pp. 69-77, 2019.

[8] H. Bergman, J. Daugirdas, T. Ing et al., Handbook of Dialysis, Lippincott, Williams \& Wilkins, Philadelphia, PA, USA, 2001.

[9] A. Kumar, A. Cage, and R. Dhar, "Dialysis-induced worsening of cerebral edema in intracranial hemorrhage: a case series and clinical perspective," Neurocritical Care, vol. 22, no. 2, pp. 283-287, 2014. 
[10] H. E. Hinson, D. Stein, and K. N. Sheth, "Hypertonic saline and mannitol therapy in critical care neurology," Journal of Intensive Care Medicine, vol. 28, no. 1, pp. 3-11, 2011.

[11] D. Zepeda-Orozco and R. Quigley, "Dialysis disequilibrium syndrome," Pediatric Nephrology, vol. 27, no. 12, pp. 22052211, 2012. 